Preprint n.

Dipartimento di Fisica

Università di Roma "La Sapienza"

I.N.F.N. - Sezione di Roma

\title{
ABOUT THE OVERLAP DISTRIBUTION IN MEAN FIELD SPIN GLASS MODELS $\ddagger$
}

\author{
by \\ Francesco Guerra \\ Dipartimento di Fisica, Università di Roma "La Sapienza", \\ and Istituto Nazionale di Fisica Nucleare, Sezione di Roma, \\ Piazzale Aldo Moro, 2, I-00185 Roma, Italy. \\ e-mail guerra@roma1.infn.it \\ WWW http://romagtc.roma1.infn.it/
}

November 1995

\begin{abstract}
.
We continue our presentation of mathematically rigorous results about the SherringtonKirkpatrick mean field spin glass model. Here we establish some properties of the distribution of overlaps between real replicas. They are in full agreement with the Parisi accepted picture of spontaneous replica symmetry breaking. As a byproduct, we show that the selfaveraging of the Edwards-Anderson fluctuating order parameter, with respect to the external quenched noise, implies that the overlap distribution is given by the Sherrington-Kirkpatrick replica symmetric Ansatz. This extends previous results of Pastur and Shcherbina. Finally, we show how to generalize our results to realistic short range spin glass models.
\end{abstract}

Dedicated to the memory of Hiroomi Umezawa

$\ddagger$ Research supported in part by MURST (Italian Minister of University and Scientific and Technological Research) and INFN (Italian National Institute for Nuclear Physics). 


\section{INTRODUCTION.}

The physical content of the Sherrington-Kirkpatrick mean field spin glass model $[1,2]$ is fully contained in the probabilistic distribution of overlaps between replicas of the system. In the approximate Sherrington-Kirkpatrick Ansatz [1], this distribution is concentrated on a numerical order parameter, while in the more complete Parisi treatment [2], based on replica symmetry breaking, the distribution shows ultrametric features and can be expressed through a functional order parameter (see also $[3,4,5]$ ).

We have found a general very elementary method able to generate rigorous relations between replica averages, also in the infinite volume limit. The method is based on simple analysis of fluctuations. The purpose of this paper is to show some applications of this method.

If we consider four replicas, dubbed $1,2,3,4$, as defined in the following, then the ultrametric picture gives a very simple statement about the joint distributions of the overlaps $q_{12}, q_{23}$ and $q_{12}, q_{34}$, respectively. Roughly speaking, for the joint distribution of $q_{12}, q_{23}$, it says that the distribution of $q_{23}$ is such that $q_{23}$ is equal to $q_{12}$ with probability one half, and independent from $q_{12}$ with the same probability one half. Notice that the statement does not depend on the precise form of the Parisi functional order parameter, neither on the temperature. In this case the overlaps $q_{12}, q_{23}$ have one replica in common. If we consider the overlaps $q_{12}, q_{34}$, among disjoint replicas, then the statement is the same as before, with the probabilities one half - one half replaced by one third - two thirdies, respectively. The fact that two overlaps, as $q_{12}, q_{34}$, related to disjoint replicas, are not completely independent is very peculiar to the ultrametric picture, and is a consequence of the important phenomenon of the absence of selfaveraging for some fluctuating observables.

As an example of application of our general method, we will give a very simple rigorous proof for the following equalities among overlap averages, direct consequences of the previous ultrametric statements,

$$
\begin{aligned}
& \left\langle q_{12}^{2} q_{23}^{2}\right\rangle=\frac{1}{2}\left\langle q_{12}^{4}\right\rangle+\frac{1}{2}\left\langle q_{12}^{2}\right\rangle^{2}, \\
& \left\langle q_{12}^{2} q_{34}^{2}\right\rangle=\frac{1}{3}\left\langle q_{12}^{4}\right\rangle+\frac{2}{3}\left\langle q_{12}^{2}\right\rangle^{2} .
\end{aligned}
$$

In a recent paper [6], Marinari, Parisi, Ruiz-Lorenzo and Ritort have found, among other things, convincing numerical evidence, based on computer simulations on large systems, that these equalities hold also for spin glass systems with short range interactions. This provides support to the idea that some relevant features of the ultrametric picture survive also in the transition from the mean field model to more realistic disordered short range interactions (but see also [7]).

While it is true that the overlap distributions do not reproduce the full physical content of the short range models, nevertheless it is interesting to notice that our methods are able to establish equalities $(1,2)$, also in the short range case, modulo some technical assumptions about the infinite volume limit, in full agreement with the computer simulations.

As a byproduct of our analysis, we can also establish rigorously that selfaveraging of the Edwards-Anderson fluctuating order parameter implies that the overlap distribution is 
trivially given by the Sherrington-Kirkpatrick replica symmetric Ansatz, through a simple numerical order parameter. The precise statements are presented in the following, but we can see immediately from (2) that selfaveraging of the fluctuating order parameter would imply, in the infinite volume limit, that

$$
\left\langle q_{12}^{2} q_{34}^{2}\right\rangle=\left\langle q_{12}^{2}\right\rangle\left\langle q_{34}^{2}\right\rangle=\left\langle q_{12}^{2}\right\rangle^{2}
$$

and therefore

$$
\left\langle q_{12}^{4}\right\rangle=\left\langle q_{12}^{2}\right\rangle^{2}
$$

In this case, all overlaps would be constant.

It is expected that for high temperatures and/or high values of the external field the model exhibits selfaveraging (see for example [8] and [3]), but for zero external field and low temperatures our result shows that there can not be selfaveraging, because the SherringtonKirkpatrick replica symmetric Ansatz then becomes incompatible with positivity of the entropy.

Our results, about lack of selfaveraging, complement previous similar results by Pastur and Shcherbina [9] and Shcherbina [10], where they consider fluctuating order parameters coming from the response of the system to suitably chosen external random fields.

The paper is organized as follows. In Section 2, we recall the basic definitions of the mean field spin glass model, and introduce the overlap parameters and their averages, in connection with the thermodynamic observables of the theory. In particular, we show how to express the specific internal energy and its temperature derivative, and the quadratic fluctuations for the internal energy and the order parameter in terms of overlap averages. In Section 3, we exploit simple positivity properties in order to prove some ultrametric inequalities. Then, we show that selfaveraging for the fluctuating order parameter implies that all overlaps are constant, according to the replica symmetric Ansatz. Section 4 is devoted to the proof of the metric equalities. We will exploit the properties of selfaveraging for the specific free energy and the internal energy. Finally, Section 5 gives some conclusions and outlook for future developments.

In conclusion, useful conversations with Roberto D'Autilia, Enzo Marinari, Giorgio Parisi and Masha Shcherbina are gratefully acknowledged. We would like to thank also Charles Newman and Dan Stein for making available Ref. [7] before publication, and useful correspondence. 


\section{OVERLAP DISTRIBUTIONS.}

The mean field spin glass model is defined on sites $i=1,2, \ldots, N$, to which Ising spin variables $\sigma_{i}= \pm 1$ are attached. The external quenched disorder is given by the $N(N-1) / 2$ independent and identical distributed random variables $J_{i j}$, defined for each couple of sites. For the sake of definiteness, we assume each $J_{i j}$ to be a centered unit Gaussian with averages

$$
E\left(J_{i j}\right)=0, \quad E\left(J_{i j}^{2}\right)=1 .
$$

The Hamiltonian of the model is given by

$$
H_{N}(\sigma, J)=-\frac{1}{\sqrt{N}} \sum_{(i, j)} J_{i j} \sigma_{i} \sigma_{j}
$$

where the sum, extending to all spin couples, and the normalizing factor $1 / \sqrt{N}$ are typical of the mean field character of the model.

For a given inverse temperature $\beta$, we introduce the partition function $Z_{N}(\beta, J)$, the free energy per site $f_{N}(\beta, J)$, the internal energy per site $u_{N}(\beta, J)$, and the Boltzmann state $\omega_{J}$, according to the definitions

$$
\begin{gathered}
Z_{N}(\beta, J)=\sum_{\sigma_{1} \ldots \sigma_{N}} \exp \left(-\beta H_{N}(\sigma, J)\right), \\
-\beta f_{N}(\beta, J)=N^{-1} \log Z_{N}(\beta, J), \\
\omega_{J}(A)=Z_{N}(\beta, J)^{-1} \sum_{\sigma_{1} \ldots \sigma_{N}} A \exp \left(-\beta H_{N}(\sigma, J)\right), \\
u_{N}(\beta, J)=N^{-1} \omega_{J}\left(H_{N}(\sigma, J)\right)=\partial_{\beta}\left(\beta f_{N}(\beta, J)\right),
\end{gathered}
$$

where $A$ is a generic function of the $\sigma$ 's. In the notation $\omega_{J}$, we have stressed the dependence of the Boltzmann state on the external noise $J$, but, of course, there is also a dependence on $\beta$ and $N$.

We are interested in the thermodynamic limit $N \rightarrow \infty$.

Let us consider a generic number $s$ of independent copies (replicas) of the system, characterized by the Boltzmann variables $\sigma_{i}^{(1)}, \sigma_{i}^{(2)}, \ldots$, distributed according to the product state

$$
\Omega_{J}=\omega_{J}^{(1)} \omega_{J}^{(2)} \ldots \omega_{J}^{(s)},
$$

where all $\omega_{J}^{(\alpha)}$ act on each one $\sigma_{i}^{(\alpha)}$,s, and are subject to the same sample $J$ of the external noise.

The overlaps between two replicas $\alpha, \beta$ are defined according to

$$
Q_{\alpha \beta}\left(\sigma^{(\alpha)}, \sigma^{(\beta)}\right)=\frac{1}{N} \sum_{i} \sigma_{i}^{(\alpha)} \sigma_{i}^{(\beta)}
$$

with the obvious bounds

$$
-1 \leq Q_{\alpha \beta} \leq 1
$$


For a generic smooth function $F$ of the overlaps, we define the averages

$$
E \Omega_{J}\left(F\left(Q_{12}, Q_{13}, \ldots\right)\right),
$$

where $\Omega_{J}$ acts on the $\sigma$ variables, and $E$ is the average with respect to the external noise $J$. We introduce also random variables $q_{12}, q_{13}, \ldots$, through the definition of their averages

$$
\left\langle F\left(q_{12}, q_{13}, \ldots\right)\right\rangle=E \Omega_{J}\left(F\left(Q_{12}, Q_{13}, \ldots\right)\right) .
$$

We have made a careful distinction in notations. In fact, \langle\rangle contains both Boltzmann averages $\Omega_{J}$ and noise averages $E$.

It is important to remark that the noise average $E$ introduces correlations between different groups of replicas. For example, before the $E$ average, we have the factorization

$$
\Omega_{J}\left(q_{12}^{2} q_{34}^{2}\right)=\left(\omega_{J}^{(1)} \omega_{J}^{(2)}\right)\left(q_{12}^{2}\right)\left(\omega_{J}^{(3)} \omega_{J}^{(4)}\right)\left(q_{34}^{2}\right) .
$$

But after the $E$ average we have in general

$$
\left\langle q_{12}^{2} q_{34}^{2}\right\rangle \neq\left\langle q_{12}^{2}\right\rangle\left\langle q_{34}^{2}\right\rangle .
$$

The \langle\rangle averages are obviously invariant under permutations of the replicas, so that in particular

$$
\left\langle q_{12}^{2}\right\rangle=\left\langle q_{34}^{2}\right\rangle .
$$

Moreover, they are invariant under gauge transformations

$$
q_{\alpha \beta} \rightarrow \epsilon_{\alpha} \epsilon_{\beta} q_{\alpha \beta},
$$

where $\epsilon_{\alpha}= \pm 1$. This is an easy consequence of the fact that each $\omega_{J}^{(\alpha)}$ is an even state on the respective $\sigma$ 's.

There are important consequences from gauge invariance. First of all, polynomials in the overlaps, which are not gauge invariant, have zero mean. Moreover, the distributions are uniquely defined by their restrictions to the case where all values of the $q$ 's are nonnegative. The general case, in all regions of possible values for the $q$ 's, is easily reconstructed using gauge invariance.

Overlap distributions carry the whole physical content of the theory. In fact, the averages of the physical observables with respect to the external noise can be expressed through averages involving overlap functions. As an example, we give the following statements involving the internal energy, its $\beta$ derivative and its quadratic fluctuations.

Theorem 1. The noise average of the internal energy is given through an overlap average of the type

$$
E\left(u_{N}(\beta, J)\right)=N^{-1} E\left(\omega_{J}\left(H_{N}(\sigma, J)\right)\right)=-\frac{1}{2} \beta\left(1-\left\langle q_{12}^{2}\right\rangle\right) .
$$

Theorem 2. The $\beta$ derivative of the noise average of the internal energy is expressed as

$$
\begin{aligned}
& \partial_{\beta} E\left(u_{N}(\beta, J)\right)=-N^{-1} E\left(\omega_{J}\left(H_{N}^{2}\right)-\omega_{J}^{2}\left(H_{N}\right)\right) \\
& =-\frac{1}{2}\left(1-\left\langle q_{12}^{2}\right\rangle\right)+\frac{\beta^{2}}{2} N\left(\left\langle q_{12}^{4}\right\rangle-4\left\langle q_{12}^{2} q_{23}^{2}\right\rangle+3\left\langle q_{12}^{2} q_{34}^{2}\right\rangle\right) .
\end{aligned}
$$


Theorem 3. For the quadratic fluctuation of the internal energy we have

$$
\begin{aligned}
& E\left(u_{N}^{2}(\beta, J)\right)-\left(E u_{N}(\beta, J)\right)^{2}=N^{-2}\left(E\left(\omega_{J}^{2}\left(H_{N}\right)\right)-\left(E \omega_{J}\left(H_{N}\right)\right)^{2}\right) \\
& =-\frac{1}{2 N}\left\langle q_{12}^{2}\right\rangle-\frac{1}{2 N^{2}}+\frac{3}{2} \beta^{2}\left(\left\langle q_{12}^{2} q_{34}^{2}\right\rangle-\left\langle q_{12}^{2} q_{23}^{2}\right\rangle\right)+\frac{1}{4} \beta^{2}\left(\left\langle q_{12}^{4}\right\rangle-\left\langle q_{12}^{2}\right\rangle^{2}\right) .
\end{aligned}
$$

The proof of these results is very straigthforward, but it involves long calculations. The main ingredient is nothing but integration by part with respect to the external noise. In fact, for a unit Gaussian $J$ and a smooth function $F$, we have

$$
E(J F(J))=E\left(\frac{\partial}{\partial J} F(J)\right) .
$$

As an example, we recall the proof of (20) (see [2], and [3]). Starting from the definitions $(6,9,10,12)$, we can write the following chain of equalities

$$
\begin{aligned}
& -E\left(u_{N}(\beta, J)\right)=-N^{-1} E\left(\omega_{J}\left(H_{N}(\sigma, J)\right)\right)=\frac{1}{N \sqrt{N}} \sum_{(i, j)} E\left(J_{i j} \omega_{J}\left(\sigma_{i} \sigma_{j}\right)\right) \\
& =\frac{\beta^{2}}{N^{2}} \sum_{(i, j)}\left(1-E\left(\omega_{J}^{2}\left(\sigma_{i} \sigma_{j}\right)\right)\right)=\frac{\beta^{2}}{2 N^{2}} \sum_{i, j}\left(1-E\left(\omega_{J}^{2}\left(\sigma_{i} \sigma_{j}\right)\right)\right) \\
& =\frac{\beta^{2}}{2}\left(1-\frac{1}{N^{2}} \sum_{i, j} E\left(\omega_{J}^{(1)}\left(\sigma_{i}^{(1)} \sigma_{j}^{(1)}\right) \omega_{J}^{(2)}\left(\sigma_{i}^{(2)} \sigma_{j}^{(2)}\right)\right)\right) \\
& =\frac{1}{2} \beta\left(1-\left\langle q_{12}^{2}\right\rangle\right) .
\end{aligned}
$$

Notice that we have changed the sum over all couples $(i, j)$ into one half of the sum over all $i$ and $j$, by taking into account that the diagonal elements, corresponding to $i=j$, do not give any contribution. Formulae (21) and (22) are established in a similar way. They involve a double integration by parts, since the Hamiltonian $H_{N}$ appears quadratically.

We can also introduce the fluctuating order parameter

$$
M_{N}^{2}(\beta, J)=\frac{1}{N^{2}} \sum_{i, j} \omega_{J}^{2}\left(\sigma_{i} \sigma_{j}\right)=\Omega_{J}\left(Q_{12}^{2}\right),
$$

and notice that

$$
E\left(M_{N}^{2}(\beta, J)\right)=\left\langle q_{12}^{2}\right\rangle, \quad E\left(M_{N}^{4}(\beta, J)\right)=\left\langle q_{12}^{2} q_{34}^{2}\right\rangle .
$$




\section{CONSEQUENCES OF SELFAVERAGING.}

Since (21) is clearly nonpositive, and (22) nonnegative, we have immediately the following bounds

$$
\begin{gathered}
\left\langle q_{12}^{2} q_{23}^{2}\right\rangle \geq \frac{1}{4}\left\langle q_{12}^{4}\right\rangle+\frac{3}{4}\left\langle q_{12}^{2} q_{34}^{2}\right\rangle+O\left(\frac{1}{N}\right), \\
\left\langle q_{12}^{2} q_{23}^{2}\right\rangle \leq\left\langle q_{12}^{2} q_{34}^{2}\right\rangle+\frac{1}{6}\left(\left\langle q_{12}^{4}\right\rangle-\left\langle q_{12}^{2}\right\rangle^{2}\right)+O\left(\frac{1}{N}\right) .
\end{gathered}
$$

Now we are ready to establish

Theorem 4. The following estimates hold

$$
\begin{aligned}
& \left\langle q_{12}^{2} q_{23}^{2}\right\rangle \geq \frac{1}{2}\left\langle q_{12}^{4}\right\rangle+\frac{1}{2}\left\langle q_{12}^{2}\right\rangle^{2}+O\left(\frac{1}{N}\right), \\
& \left\langle q_{12}^{2} q_{34}^{2}\right\rangle \geq \frac{1}{3}\left\langle q_{12}^{4}\right\rangle+\frac{2}{3}\left\langle q_{12}^{2}\right\rangle^{2}+O\left(\frac{1}{N}\right) .
\end{aligned}
$$

In order to prove (30), it is sufficient to eliminate $\left\langle q_{12}^{2} q_{23}^{2}\right\rangle$ between (27) and (28). Then, (29) follows from the elimination of $\left\langle q_{12}^{2} q_{34}^{2}\right\rangle$ between (30) and (27).

An obvious consequence of $(29,30)$ is the following

Theorem 5. In the limit for $N \rightarrow \infty$ we have

$$
\begin{aligned}
& \liminf _{N \rightarrow \infty}\left(\left\langle q_{12}^{2} q_{23}^{2}\right\rangle-\frac{1}{2}\left\langle q_{12}^{4}\right\rangle-\frac{1}{2}\left\langle q_{12}^{2}\right\rangle^{2}\right) \geq 0, \\
& \left.\liminf _{N \rightarrow \infty}\left(\left\langle q_{12}^{2} q_{34}^{2}\right\rangle-\frac{1}{3}\left\langle q_{12}^{4}\right\rangle-\frac{2}{3}\left\langle q_{12}^{2}\right\rangle^{2}\right)\right) \geq 0 .
\end{aligned}
$$

Notice that the results of Theorem 4 and Theorem 5 rely only on positivity properties for the expressions $(21,22)$.

Finally, we can connect the quadratic fluctuations for the order parameter defined in (25) with the quadratic fluctuations for the overlap $q_{12}^{2}$. In fact, from (26) and (30), we immediately have

Theorem 6. The following estimate holds

$$
0 \leq\left\langle q_{12}^{4}\right\rangle-\left\langle q_{12}^{2}\right\rangle^{2} \leq 3\left(E\left(M_{N}^{4}(\beta, J)\right)-E^{2}\left(M_{N}^{2}(\beta, J)\right)\right)+O\left(\frac{1}{N}\right) .
$$

Therefore, we have a simple proof of the following very important result

Theorem 7. Assume that the order parameter $M_{N}^{2}$ is selfaveraging in the infinite volume limit, in the sense that for $N \rightarrow \infty$ along some subsequence we have

$$
\lim _{N \rightarrow \infty}\left(E\left(M_{N}^{4}(\beta, J)\right)-E^{2}\left(M_{N}^{2}(\beta, J)\right)\right)=0 .
$$

Then, along the same subsequence

$$
\lim _{N \rightarrow \infty}\left(\left\langle q_{12}^{4}\right\rangle-\left\langle q_{12}^{2}\right\rangle^{2}\right)=0
$$


If, moreover, we chose $N \rightarrow \infty$ along a subsequence such that

$$
\lim _{N \rightarrow \infty}\left\langle q_{12}^{2}\right\rangle=\bar{q}^{2}
$$

where for example

$$
\bar{q}^{2}=\limsup _{N \rightarrow \infty}\left\langle q_{12}^{2}\right\rangle,
$$

then along the same subsequence we have for any gauge invariant function $F$

$$
\lim _{N \rightarrow \infty}\left\langle F\left(q_{12}, q_{13}, \ldots\right)\right\rangle=F(\bar{q}, \bar{q}, \ldots),
$$

so that the overlaps have the Sherrington-Kirkpatrick replica symmetric form.

Clearly, (35) follows from (38) through (33), while (38) is a simple consequence of the absence of dispersion for the overlaps.

This result complements previous results by Pastur and Shcherbina [9], and Shcherbina [10], where they prove that quadratic selfaveraging for some order parameters, coming from the response of the system to random external fields, implies the Sherrington-Kirkpatrick form for the solution of the model.

\section{THE ULTRAMETRIC EQUALITIES.}

In [9], Pastur and Shcherbina have proven the selfaveraging of the free energy per site in quadratic mean, in the infinite volume limit. In fact, by a mild improvement of their methods, one can easily show [11] the following

Theorem 8. The quadratic fluctuation of the free energy per site can be estimated as follows

$$
E\left(\left(N^{-1} \log Z_{N}(\beta, J)\right)^{2}\right)-\left(E\left(N^{-1} \log Z_{N}(\beta, J)\right)\right)^{2} \leq \frac{\beta^{2}}{2 N}\left\langle q_{12}^{2}\right\rangle+O\left(\frac{1}{N^{2}}\right) .
$$

By using standard techniques of statistical mechanics, one can easily derive statements about the quadratic fluctuation of the internal energy. A precise formulation is the following.

Let $N$ go to infinity along a subsequence such that the average specific energy is convergent

$$
\left.\lim _{N \rightarrow \infty} N^{-1} E \log Z_{N}(\beta, J)\right)=-\beta f(\beta) .
$$

Due to convexity in $\beta$ the subsequence can be chosen without exploiting compacteness or the axiom of choice [11], as for any subsequence in this paper, by working for example always with limsup's for a denumerable dense set of $\beta$ 's.

Since the function $-\beta f(\beta)$ is convex in $\beta$, there exist the left and right $\beta$ derivatives $u_{( \pm)}(\beta)$, and, moreover, they are equal for all values of $\beta$, with the possible exclusion of a set of zero Lebesgue measure. Then, we have [11] 
Theorem 9. For $N$ going to infinity, along the chosen subsequence, the quadratic fluctuation of the specific internal energy satisfies

$$
\limsup _{N \rightarrow \infty}\left(E\left(u_{N}^{2}(\beta, J)\right)-\left(E u_{N}(\beta, J)\right)^{2}\right) \leq \frac{1}{4}\left(u_{(+)}(\beta)-u_{(-)}(\beta)\right)^{2} .
$$

Therefore, we have quadratic selfaveraging for almost all values of $\beta$.

For a complete detailed proof of Theorem 8 and Theorem 9 , we refer to our forthcoming review paper [11].

¿From now on, we let $N \rightarrow \infty$ along subsequences such that not only the specific free energy but also the averages $\left\langle q_{12}^{2}\right\rangle,\left\langle q_{12}^{4}\right\rangle,\left\langle q_{12}^{2} q_{23}^{2}\right\rangle,\left\langle q_{12}^{2} q_{34}^{2}\right\rangle$ have well definite limits.

An immediate consequence of Theorem 3 and Theorem 9 is the following

Theorem 10. In the limit $N \rightarrow \infty$ along the stated subsequence, for almost all values of $\beta$, we have

$$
\left\langle q_{12}^{2} q_{34}^{2}\right\rangle-\left\langle q_{12}^{2} q_{23}^{2}\right\rangle=\frac{1}{6}\left(\left\langle q_{12}^{4}\right\rangle-\left\langle q_{12}^{2}\right\rangle^{2}\right) .
$$

Further information can be gathered from $(20,21)$. In fact, at fixed $N$, by taking the $\beta$ derivative in (20), and by exploiting (21), we have

$$
-\frac{1}{2}+\frac{1}{2} \beta \partial_{\beta}\left\langle q_{12}^{2}\right\rangle=\frac{\beta^{2}}{2} N A_{N}^{2}(\beta),
$$

where we have defined the nonnegative $A_{N}^{2}(\beta)$ through

$$
A_{N}^{2}(\beta)=\left\langle q_{12}^{4}\right\rangle-4\left\langle q_{12}^{2} q_{23}^{2}\right\rangle+3\left\langle q_{12}^{2} q_{34}^{2}\right\rangle-\frac{1}{N \beta^{2}}\left(1-\left\langle q_{12}^{2}\right\rangle\right) .
$$

Let us integrate (43) in $d \beta^{2}$ on the generic interval $\left[\beta_{1}^{2}, \beta_{2}^{2}\right]$, with $0<\beta_{1}^{2}<\beta_{2}^{2}$. We have

$$
\int_{\beta_{1}^{2}}^{\beta_{2}^{2}} A_{N}^{2}(\beta) d \beta^{2}=\frac{2}{N}\left(\left\langle q_{12}^{2}\right\rangle_{\beta_{2}^{2}}-\left\langle q_{12}^{2}\right\rangle_{\beta_{1}^{2}}\right)-\frac{1}{N} \log \frac{\beta_{2}^{2}}{\beta_{1}^{2}} .
$$

Therefore, we have that $A_{N}^{2}(\beta)$ converges to zero in quadratic mean in any $\beta^{2}$ interval. We can extract a subsequence such that $A_{N}^{2}(\beta)$ converges to zero, for all values of $\beta$, by excluding a set of zero Lebesgue measure. By recalling the definition (44), we then arrive at

Theorem 11. In the limit $N \rightarrow \infty$ along the stated subsequence, for almost all values of $\beta$, we have

$$
\left\langle q_{12}^{4}\right\rangle-4\left\langle q_{12}^{2} q_{23}^{2}\right\rangle+3\left\langle q_{12}^{2} q_{34}^{2}\right\rangle=0
$$

By collecting all results of Theorems 10 and 11, we can establish the following equalities, in full agreement with the Parisi ultrametric solution of the mean field spin glass model.

Theorem 12. For almost all values of $\beta$, and for $N$ going to infinity along a chosen subsequence, we have in the limit

$$
\left\langle q_{12}^{2} q_{23}^{2}\right\rangle=\frac{1}{2}\left\langle q_{12}^{4}\right\rangle+\frac{1}{2}\left\langle q_{12}^{2}\right\rangle^{2},
$$




$$
\left\langle q_{12}^{2} q_{34}^{2}\right\rangle=\frac{1}{3}\left\langle q_{12}^{4}\right\rangle+\frac{2}{3}\left\langle q_{12}^{2}\right\rangle^{2} .
$$

Therefore, we have proven the main result of this paper. The possible restrictions on the values of $\beta$ and the limiting subsequences are a consequence of the method of proof. In fact, we believe that all results are true for any value of $\beta$ and $N$ going to infinity without restrictions. But we would need a better control on the limits.

It is immediate to generalize the results of Theorem 12 to models with short range interaction. In fact, we can add to the interaction Hamiltonian of the short range model a small mean field interaction of the type $\lambda H_{N}$, with $H_{N}$ defined as in (6). By taking $\lambda \rightarrow 0$, after the infinite volume limit, we still have $(47,48)$. The general validity of $(47,48)$ is also confirmed by numerical simulations [6].

\section{CONCLUSIONS AND OUTLOOK FOR FUTURE DEVELOPMENTS.}

In conclusion, we can see that all results explained in this paper rely on very elementary arguments, based essentially on positivity and convexity. These results are easily generalized.

For example, we can add a small interaction of the form

$$
\lambda \sum_{i} J_{i} \sigma_{i}
$$

where the $J_{i}$ are independent centered unit Gaussian external noises. By repeating all arguments in this paper, we immediately establish equalities of the type

$$
\begin{aligned}
& \left\langle q_{12} q_{23}\right\rangle=\frac{1}{2}\left\langle q_{12}^{2}\right\rangle+\frac{1}{2}\left\langle q_{12}\right\rangle^{2}, \\
& \left\langle q_{12} q_{34}\right\rangle=\frac{1}{3}\left\langle q_{12}^{2}\right\rangle+\frac{2}{3}\left\langle q_{12}\right\rangle^{2} .
\end{aligned}
$$

In a forthcoming paper, we will show other applications of this method. 


\section{REFERENCES}

[1] D. Sherrington and S. Kirkpatrick: Solvable model of a spin glass, Phys. Rev. Lett., 35, 1792 (1975).

[2] M. Mézard, G. Parisi, and M. A. Virasoro: Spin Glass Theory and Beyond, World Scientific, Singapore, 1987, and reprints included there.

[3] F. Guerra: Fluctuations and Thermodynamic Variables in Mean Field Spin Glass Models, in: Stochastic Processes, Physics and Geometry, S. Albeverio et al., eds, World Scientific, Singapore, 1995.

[4] F. Guerra: Functional Order Parameters for the Quenched Free Energy in Mean Field Spin Glass Models, Preprint, Rome, 1992, in press.

[5] F. Guerra: The Cavity Method in the Mean Field Spin Glass Model. Functional Representations of Thermodynamic Variables, in: Advances in Dynamical Systems and Quantum Physics, S. Albeverio et al., eds, World Scientific, Singapore, 1995.

[6] E. Marinari, G. Parisi, J. Ruiz-Lorenzo and F. Ritort, Numerical Evidence for Spontaneously Broken Replica Symmetry in 3D Spin Glasses, to appear.

[7] C. Newman and D. Stein, Non-Mean-Field Behavior of Realistic Spin Glasses, to appear.

[8] M. V. Shcherbina, in preparation.

[9] L. A. Pastur and M. V. Shcherbina: The Absence of Self-Averaging of the Order Parameter in the Sherrington-Kirkpatrick Model, J. Stat. Phys., 62, 1 (1991).

[10] M. V. Scherbina: More about Absence of Selfaverageness of the Order Parameter in the Sherrington-Kirkpatrick Model, CARR Reports in Mathematical Physics, n. 3/91, Department of Mathematics, University of Rome "La Sapienza", 1991.

[11] F. Guerra: On the mean field spin glass model, in preparation. 